\title{
THE GEOMETRIC STRUCTURES OF THE WEIBULL DISTRIBUTION MANIFOLD AND THE GENERALIZED EXPONENTIAL DISTRIBUTION MANIFOLD
}

\author{
LIMEI CAO, HUAFEI SUN AND XIAOJIE WANG
}

\begin{abstract}
Investigating the geometric structures of the distribution manifolds is a basic task in information geometry. However, by so far, most works are on the distribution manifolds of exponential family. In this paper, we investigate two non-exponential distribution manifolds - the Weibull distribution manifold and the generalized exponential distribution manifold. Then we obtain their geometric structures.
\end{abstract}

\section{Introduction}

Information geometry provides a new perspective from geometry to investigate a wide variety of domains such as statistics, stochastic processes, neural networks, cybernetics, cryptology etc.. Because the probability distribution is the fundamental element over such fields, investigating the geometric structure of distribution manifolds is a basic work for information geometry[1]. Amari, Dodson [2,4] and other authors studied the distributions of exponential families and gave the geometric structures for some distribution manifolds. Especially, the geometric structures of the normal distribution manifold, the Gamma distribution manifold, the Frund distribution manifold and the Beta distribution manifold were obtained by some authors. Meanwhile the Weibull distribution and the generalized exponential distribution are two famous distributions with wide applications. However, the Weibull distribution and the generalized exponential distribution are not the exponential families, because they can not be written as the exponential forms. In other words, we can not use the potential function to calculate their quantities of geometry. In this paper, we investigate the Weibull distribution manifold and the generalized exponential distribution manifold without the help of potential function, and obtain their Ricci curvatures and Gaussian curvatures.

Received July 25, 2006; revised December 20, 2006.

2000 Mathematics Subject Classification. 53B20, 62H10.

Key words and phrases. Information geometry, Weibull distribution, generalized exponential distribution. 


\section{The Weibull distribution manifold}

The set

$$
S=\left\{p(x) \mid p(x)=\left[\frac{b}{a}\left(\frac{x}{a}\right)^{b-1}\right] \exp \left\{-\left(\frac{x}{a}\right)^{b}\right\}, x \in[0,+\infty),(a, b) \in R^{+} \times R^{+}\right\}
$$

is called the Weibull distribution manifold, where

$$
p(x)=\left[\frac{b}{a}\left(\frac{x}{a}\right)^{b-1}\right] \exp \left\{-\left(\frac{x}{a}\right)^{b}\right\}
$$

is the probability density function of the Weibull distribution. And $(a, b)$ plays the role of the coordinate system.

From (2.1), we get the log-likelihood function

$$
\log p(x)=-a^{-b} x^{b}+\log b+(b-1) \log x-b \log a .
$$

Set $l(x)=\log p(x)$. Taking the coordinate $\left(\theta_{1}, \theta_{2}\right)=(a, b)$ and setting $\partial_{i} l=\frac{\partial}{\partial \theta_{i}} l(x)$, from (2.2), we get

$$
\begin{aligned}
& \partial_{1} \partial_{1} l=-\left(b^{2}+b\right) a^{-b-2} x^{b}+\frac{b}{a^{2}}, \\
& \partial_{1} \partial_{2} l=\partial_{2} \partial_{1} l=x^{b} a^{-b-1}+b a^{-b-1} x^{b} \log x-b a^{-b-1} \log a^{b} x, \\
& \partial_{2} \partial_{2} l=-a^{-b} x^{b}\left(\log \frac{x}{a}\right)^{2}-\frac{1}{b^{2}} .
\end{aligned}
$$

By direct calculation, we obtain the following expectations

$$
\begin{aligned}
& E\left[x^{b}\right]=a^{b}, \\
& E\left[x^{b} \log x\right]=\frac{a^{b}}{b}(1-\xi)+a^{b} \log a, \\
& E\left[x^{b}\left(\log \frac{x}{a}\right)^{2}\right]=\frac{a^{b}}{b^{2}}\left(\xi^{2}-2 \xi+\frac{\pi^{2}}{6}\right),
\end{aligned}
$$

where $\xi$ is the Euler's constant and $E(f)$ means the expectation of $f$.

And further

$$
\begin{aligned}
& E\left[\partial_{1} \partial_{1} l\right]=-\frac{b^{2}}{a^{2}}, \\
& E\left[\partial_{1} \partial_{2} l\right]=E\left[\partial_{2} \partial_{1} l\right]=\frac{1-\xi}{a}, \\
& E\left[\partial_{2} \partial_{2} l\right]=-\frac{\left(\xi^{2}-2 \xi+\frac{\pi^{2}}{6}+1\right)}{b^{2}} .
\end{aligned}
$$

The Fisher information matrix $\left(g_{i j}\right)$ is defined by

$$
g_{i j}=E\left[\partial_{i} l \partial_{j} l\right]
$$

Therefore, we obtain the following result. 
Proposition 2.1. $\left(g_{i j}\right)$ and its inverse $\left(g^{i j}\right)$ are given by

$$
\left(g_{i j}\right)=\left(\begin{array}{cc}
\frac{b^{2}}{a^{2}} & \frac{\xi-1}{a} \\
\frac{\xi-1}{a} & \frac{\xi^{2}-2 \xi+\frac{\pi^{2}}{6}+1}{b^{2}}
\end{array}\right)
$$

and

$$
\left(g^{i j}\right)=\left(\begin{array}{cc}
\frac{6 a^{2}\left(\xi^{2}-2 \xi+\frac{\pi^{2}}{6}+1\right)}{\pi^{2} b^{2}} & \frac{6 a(1-\xi)}{\pi^{2}} \\
\frac{6 a(1-\xi)}{\pi^{2}} & \frac{6 b^{2}}{\pi^{2}}
\end{array}\right),
$$

respectively.

By proposition 2.1, we get the following result.

Proposition 2.2. The square of the arc length element and the area element are given by

$$
d s^{2}=\frac{b^{2}}{a^{2}} d a^{2}+2 \frac{\xi-1}{a} d a d b+\frac{\xi^{2}-2 \xi+\frac{\pi^{2}}{6}+1}{b^{2}} d b^{2}
$$

and

respectively.

$$
d A=\sqrt{\operatorname{det}\left(g_{i j}\right)} d a d b=\frac{\pi}{\sqrt{6} a} d a d b,
$$

Theorem 2.3. The Ricci curvatures and the Gaussian curvature of the Weibull distribution manifold are given by

$$
\begin{aligned}
& R_{11}=-\frac{6 b^{2}}{\pi^{2} a^{2}}, R_{12}=R_{21}=\frac{6(1-\xi)}{\pi^{2} a}, R_{22}=-\frac{6\left(\xi^{2}-2 \xi+\frac{\pi^{2}}{6}+1\right)}{\pi^{2} b^{2}}, \\
& K=-\frac{6}{\pi^{2}}
\end{aligned}
$$

Proof. Since

$$
\Gamma_{i j}^{k}=g^{k l}\left(\frac{\partial g_{l i}}{\partial \theta_{j}}+\frac{\partial g_{l j}}{\partial \theta_{i}}-\frac{\partial g_{i j}}{\partial \theta_{l}}\right)
$$

by proposition 2.1 , we have

$$
\begin{aligned}
& \Gamma_{11}^{1}=\frac{6\left(\xi b-b-\frac{\pi^{2}}{6}\right)}{\pi^{2} a}, \quad \Gamma_{11}^{2}=-\frac{b^{3}}{\pi^{2} a^{2}}, \\
& \Gamma_{21}^{1}=\Gamma_{12}^{1}=\frac{6\left(\xi^{2}-2 \xi+\frac{\pi^{2}}{6}+1\right)}{\pi^{2} b}, \Gamma_{21}^{2}=\Gamma_{12}^{2}=\frac{6 b(1-\xi)}{\pi^{2} a}, \\
& \Gamma_{22}^{1}=-\frac{6 a(1-\xi)\left(\xi^{2}-2 \xi+\frac{\pi^{2}}{6}+1\right)}{\pi^{2} b^{3}}, \Gamma_{22}^{2}=-\frac{6\left(\xi^{2}-2 \xi+\frac{\pi^{2}}{6}+1\right)}{\pi^{2} b} .
\end{aligned}
$$

From

$$
R_{i j k l}=\left(\partial_{j} \Gamma_{i k}^{s}-\partial_{i} \Gamma_{j k}^{s}\right) g_{s l}+\left(\Gamma_{j t l} \Gamma_{i k}^{t}-\Gamma_{i t l} \Gamma_{j k}^{t}\right),
$$


we get

$$
R_{1212}=-R_{2112}=-R_{1221}=R_{2121}=-\frac{1}{a^{2}}
$$

and

$$
R_{11}=-\frac{6 b^{2}}{\pi^{2} a^{2}}, \quad R_{12}=R_{21}=\frac{6(1-\xi)}{\pi^{2} a}, R_{22}=-\frac{6\left(\xi^{2}-2 \xi+\frac{\pi^{2}}{6}+1\right)}{\pi^{2} b^{2}} .
$$

By

$$
K=\frac{R_{1212}}{\operatorname{det}\left(g_{i j}\right)}
$$

we get the Gaussian curvature

$$
K=-\frac{6}{\pi^{2}}
$$

This completes the proof.

\section{The generalized exponential distribution manifold}

The set

$$
S=\left\{p(x) \mid p(x ; \alpha, \beta)=\alpha \beta\left(1-e^{-\beta x}\right)^{\alpha-1} e^{-\beta x}, x \in(0, \infty), \alpha \in R^{+}, \alpha \neq 1,2, \beta \in R^{+}\right\}
$$

is called the generalized exponential manifold, where

$$
p(x ; \alpha, \beta)=\alpha \beta\left(1-e^{-\beta x}\right)^{\alpha-1} e^{-\beta x}
$$

is the probability density function of the generalized exponential distribution.

From (3.1), we have the log-likelihood function

$$
\log p(x)=\log \alpha+\log \beta+(\alpha-1) \log \left(1-e^{-\beta x}\right)-\beta x .
$$

Set $l(x)=\log p(x)$, and take the coordinate $\left(\theta_{1}, \theta_{2}\right)=(\alpha, \beta)$. Similar to the previous section, we have

$$
\begin{aligned}
& \partial_{1} \partial_{1} l=-\frac{1}{\alpha^{2}}, \\
& \partial_{1} \partial_{2} l=\partial_{2} \partial_{1} l=\frac{x}{e^{\beta x}-1}, \\
& \partial_{2} \partial_{2} l=-\frac{1}{\beta^{2}}-\frac{(\alpha-1) e^{\beta x} x^{2}}{\left(e^{\beta x}-1\right)^{2}}
\end{aligned}
$$

and

$$
\begin{aligned}
& E\left[\partial_{1} \partial_{1} l\right]=-\frac{1}{\alpha^{2}}, \\
& E\left[\partial_{1} \partial_{2} l\right]=E\left[\partial_{2} \partial_{1} l\right]=\frac{H(\alpha)-1}{(\alpha-1) \beta}, \\
& E\left[\partial_{2} \partial_{2} l\right]=-\frac{1}{\beta^{2}}-\frac{\alpha}{6 \beta^{2}(\alpha-2)}\left(6 \xi(\xi-2)+\pi^{2}+6 \psi(\alpha)(2 \xi-2+\psi(\alpha))-6 \psi^{\prime}(\alpha)\right),
\end{aligned}
$$


where $\xi$ is the Euler's constant, $H(\alpha):=\sum_{n=1}^{\infty}\left(\frac{1}{n}-\frac{1}{n+\alpha}\right)$, and $\psi(\alpha):=\frac{\Gamma^{\prime}(\alpha)}{\Gamma(\alpha)}$.

Thus, we have the following proposition.

Proposition 3.1. The Fisher information matrix $\left(g_{i j}\right)$ and its inverse $\left(g^{i j}\right)$ are given by

$$
\left(g_{i j}\right)=\left(\begin{array}{cc}
\frac{1}{\alpha^{2}} & \frac{1-H(\alpha)}{(\alpha-1) \beta} \\
\frac{1-H(\alpha)}{(\alpha-1) \beta} & \frac{1}{\beta^{2}}+\frac{T(\alpha)}{\beta^{2}}
\end{array}\right),
$$

and

$$
\left(g^{i j}\right)=\left(\begin{array}{cc}
\frac{(1+T(\alpha))(\alpha-1)^{2} \alpha^{2}}{K(\alpha)} & -\frac{\beta(1-H(\alpha))(\alpha-1) \alpha^{2}}{K(\alpha)} \\
-\frac{\beta(1-H(\alpha))(\alpha-1) \alpha^{2}}{K(\alpha)} & \frac{\beta^{2}(\alpha-1)^{2}}{K(\alpha)}
\end{array}\right),
$$

where

$$
\begin{aligned}
& T(\alpha)=\frac{\alpha}{(\alpha-2)}\left((\xi-2) \xi+\frac{\pi^{2}}{6}+\psi(\alpha)(2 \xi-2+\psi(\alpha))-\psi^{\prime}(\alpha)\right), \\
& K(\alpha)=(1+T(\alpha))(\alpha-1)^{2}-(1-H(\alpha))^{2} \alpha^{2} .
\end{aligned}
$$

By proposition 2.1, we get the following result.

Proposition 3.2. The square of the arc length element and the area element are given by

$$
d s^{2}=\frac{1}{\alpha^{2}} d \alpha^{2}+2 \frac{1-H(\alpha)}{(\alpha-1) \beta} d \alpha d \beta+\left(\frac{1}{\beta^{2}}+\frac{T(\alpha)}{\beta^{2}}\right) d \beta^{2}
$$

and

$$
d A=\sqrt{\left(g_{i j}\right)} d \alpha d \beta
$$

By (2.6) and (2.7), we obtain the following proposition.

Proposition 3.3. The components of the connection are given by

$$
\begin{aligned}
& \Gamma_{11}^{1}=\frac{H^{\prime}(\alpha)(1-H(\alpha)) \alpha^{2}}{K(\alpha)}+\frac{(1-H(\alpha))^{2} \alpha^{2}}{(\alpha-1) K(\alpha)}-\frac{(1+T(\alpha))(\alpha-1)^{2}}{\alpha K(\alpha)}, \\
& \Gamma_{11}^{2}=\frac{\beta(1-H(\alpha))(\alpha-1)}{\alpha K(\alpha)}-\frac{\beta H^{\prime}(\alpha)(\alpha-1)}{K(\alpha)}-\frac{\beta(1-H(\alpha))}{K(\alpha)}, \\
& \Gamma_{12}^{1}=\Gamma_{21}^{1}=-\frac{T^{\prime}(\alpha)(1-H(\alpha))(\alpha-1) \alpha^{2}}{2 \beta K(\alpha)}, \\
& \Gamma_{12}^{2}=\Gamma_{21}^{2}=\frac{T^{\prime}(\alpha)(\alpha-1)^{2}}{2 K(\alpha)} \\
& \Gamma_{22}^{1}=-\frac{T^{\prime}(\alpha)(1+T(\alpha))(\alpha-1)^{2} \alpha^{2}}{2 \beta^{2} K(\alpha)}, \\
& \Gamma_{22}^{2}=-\frac{1}{\beta}+\frac{T^{\prime}(\alpha)(1-H(\alpha))(\alpha-1) \alpha^{2}}{2 \beta K(\alpha)} .
\end{aligned}
$$


From proposition 3.3, we get the nonzero components of the curvature tensor

$$
\begin{aligned}
R_{1212} & =-\frac{T^{\prime \prime}(\alpha)}{2 \beta^{2}}+\frac{\left(T^{\prime}(\alpha)\right)^{2}(\alpha-1)^{2}}{4 \beta^{2} K(\alpha)}+\frac{T^{\prime}(\alpha) H^{\prime}(\alpha)(1-H(\alpha)) \alpha^{2}}{2 \beta^{2} K(\alpha)} \\
& +\frac{T^{\prime}(\alpha)(1+T(\alpha))(\alpha-1)(\alpha+1)}{2 \alpha \beta^{2} K(\alpha)}+\frac{(1-H(\alpha))(\alpha-1)(1+T(\alpha))(\beta-1)}{\alpha \beta^{2} K(\alpha)} \\
& -\frac{(1+T(\alpha)) T(\alpha)(\alpha-1)}{\beta^{2} K(\alpha)}+\frac{T^{\prime}(\alpha)(1-H(\alpha))^{2} \alpha^{2}}{2 \beta^{2}(\alpha-1) K(\alpha)} .
\end{aligned}
$$

Combining (2.6) and proposition 3.3, by calculation, we obtain the following theorem.

Theorem 3.4. The Ricci curvatures and the Gaussian curvature of the generalized exponential distribution manifold are given by

$$
\begin{gathered}
R_{11}=-\frac{T^{\prime \prime}(\alpha)(\alpha-1)^{2}}{2 K(\alpha)}+\frac{\left(T^{\prime}(\alpha)\right)^{2}(\alpha-1)^{4}}{4 K^{2}(\alpha)}+\frac{T^{\prime}(\alpha) H^{\prime}(\alpha)(1-H(\alpha)) \alpha^{2}(\alpha-1)^{2}}{2 K^{2}(\alpha)} \\
+\frac{T^{\prime}(\alpha)(1+T(\alpha))(\alpha-1)^{3}(\alpha+1)}{2 \alpha K^{2}(\alpha)}+\frac{(1-H(\alpha))(1+T(\alpha))(\alpha-1)^{3}(\beta-1)}{\alpha K^{2}(\alpha)} \\
-\frac{(1+T(\alpha)) T(\alpha)(\alpha-1)^{3}}{K^{2}(\alpha)}+\frac{T^{\prime}(\alpha)(1-H(\alpha))^{2} \alpha^{2}(\alpha-1)}{2 K^{2}(\alpha)} \\
R_{12}=R_{21}=-\frac{T^{\prime \prime}(\alpha)(1-H(\alpha))(\alpha-1) \alpha^{2}}{2 \beta K(\alpha)}+\frac{\left(T^{\prime}(\alpha)\right)^{2}(1-H(\alpha))(\alpha-1)^{3} \alpha^{2}}{4 \beta K^{2}(\alpha)} \\
+\frac{T^{\prime}(\alpha) H^{\prime}(\alpha)(1-H(\alpha))^{2}(\alpha-1) \alpha^{4}}{2 \beta K^{2}(\alpha)}+\frac{T^{\prime}(\alpha)(1+T(\alpha))(\alpha-1)^{2}(\alpha+1) \alpha(1-H(\alpha))}{2 \beta K^{2}(\alpha)} \\
+\frac{(1-H(\alpha))^{2}(\alpha-1)^{2}(1+T(\alpha)) \alpha(\beta-1)}{\beta K^{2}(\alpha)}-\frac{(1+T(\alpha)) T(\alpha)(\alpha-1)^{2} \alpha^{2}(1-H(\alpha))}{\beta K^{2}(\alpha)} \\
+\frac{T^{\prime}(\alpha)(1-H(\alpha))^{3} \alpha^{4}}{2 \beta K^{2}(\alpha)}, \\
R_{22}=-\frac{T^{\prime \prime}(\alpha)(1+T(\alpha))(\alpha-1)^{2} \alpha^{2}}{2 \beta^{2} K(\alpha)}+\frac{\left(T^{\prime}(\alpha)\right)^{2}(1+T(\alpha))(\alpha-1)^{4} \alpha^{2}}{4 \beta^{2} K^{2}(\alpha)} \\
+\frac{T^{\prime}(\alpha) H^{\prime}(\alpha)(1-H(\alpha))(1+T(\alpha))(\alpha-1)^{2} \alpha^{4}}{2 \beta^{2} K^{2}(\alpha)}+\frac{T^{\prime}(\alpha)(1+T(\alpha))^{2}(\alpha-1)^{3}(\alpha+1) \alpha}{2 \beta^{2} K^{2}(\alpha)} \\
+\frac{(1-H(\alpha))(\alpha-1)^{3}(1+T(\alpha))^{2}(\beta-1) \alpha}{\beta^{2} K^{2}(\alpha)}-\frac{(1+T(\alpha))^{2} T(\alpha)(\alpha-1)^{3} \alpha^{2}}{\beta^{2} K^{2}(\alpha)} \\
+\frac{T^{\prime}(\alpha)(1-H(\alpha))^{2}(1+T(\alpha))(\alpha-1) \alpha^{4}}{2 \beta^{2} K^{2}(\alpha)},
\end{gathered}
$$




$$
\begin{aligned}
K & =-\frac{T^{\prime \prime}(\alpha)(\alpha-1)^{2} \alpha^{2}}{2 K(\alpha)}+\frac{\left(T^{\prime}(\alpha)\right)^{2}(\alpha-1)^{4} \alpha^{2}}{4 K^{2}(\alpha)}+\frac{T^{\prime}(\alpha) H^{\prime}(\alpha)(1-H(\alpha))(\alpha-1)^{2} \alpha^{4}}{2 K^{2}(\alpha)} \\
& +\frac{T^{\prime}(\alpha)(1+T(\alpha))(\alpha-1)^{3}(\alpha+1) \alpha}{2 K^{2}(\alpha)}+\frac{(1-H(\alpha))(\alpha-1)^{3}(1+T(\alpha))(\beta-1) \alpha}{K^{2}(\alpha)} \\
& -\frac{(1+T(\alpha)) T(\alpha)(\alpha-1)^{3} \alpha^{2}}{K^{2}(\alpha)}+\frac{T^{\prime}(\alpha)(1-H(\alpha))^{2}(\alpha-1) \alpha^{4}}{2 K^{2}(\alpha)} .
\end{aligned}
$$

\section{Acknowledgement}

The authors would like to thank the referees for valuable suggestions.

\section{References}

[1] S. Amari and H. Nagaoka, Methods of Information Geometry, Transactions of Mathematical Monographs, Vol.191, American Mathematical Society, 2000.

[2] S. Amari, Differential Geometrical Methods in Statistics, Springer Lecture Notes in Statistics 28, Springer-Verlag, Berlin 1985.

[3] S. Amari, Differential Geometry of a Parametric family of Invertible Linear Systems: Riemannian metric, Dual Affine Connections and Divergence, Mathematical Systems Theory, 20(1987b), 53-82.

[4] C. T. J. Dodson, Gamma manifolds and stochastic geometry. In Proceedings of the Workshop on Recent Topics in Differetial Geometry, Santiago de Compostela 16-19 July 1997. Punlic. Depto. Geometría y Topología 89(1998), 85-92.

[5] L. Cao, H. Sun and Z. Zhang, Information geometry of stock Feturn process, Journal of Beijing Institute of Technology, 27(2007), 91-94.

Department of Mathematics, Beijing Institute of Technology, Beijing 100081, China.

E-mail: caolimei@bit.edu.cn

E-mail: sunhuafei@263.net

E-mail: wangxiaojiebit@yahoo.com.cn 